AN 1MPROVED TRACTION SHOE.

JOHN JOSEPH NUTT, B.L., M.D.

Clinical Instructor in Orthopedic Surgery, Cornell University Medical College; Assistant Surgeon to the New York State Hospital for Crippled and Deformed Children. NEW YORK CITY.

The successful treatment of Shaffer's foot, invariably, and of flatfoot, frequently, depends on the elongation of the gastrocnemius muscle. Compensation in the former condition, by means of a higher heeled shoe, and splinting the deformed foot with steel arches, in the latter condition, are measures which gencrally give relief, but of themselves are not curative.

An active foot, with limitation of dorsal flexion, must suffer from the alteration of its mechanics. The changed disposition of the superincumbent weight and the abnormal points of resistance due to the restricted movements of the lever-arms, must, inevitably, cause such disturbances as result from pressure, i. e., corns, bunions, callosities, "ingrowing toe-nails" and Morton's toe and an alteration, or a tendency to an alteration, in the relations of the tarsal bones. Whether the foot becomes what is known as flat, or presents a higher arch than formerly, depends on the amount of limitation of dorsal flexion and on the strength of the plantar tissues which serve to maintain the normal medio-tarsal joint.

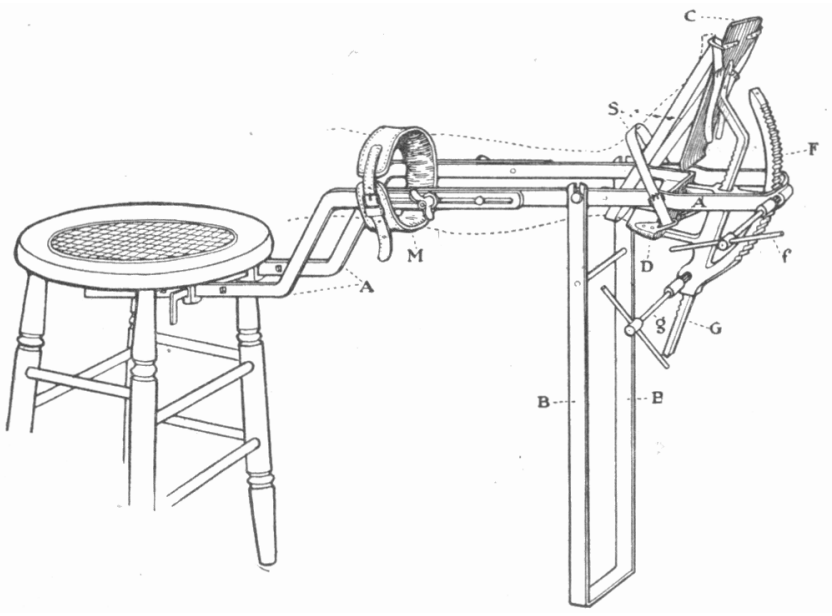

Figure 1.

Every examination of an extremity should include an examination of all its joints. Examination of the ankle joint will often determine the cause of a thigh, leg or knee pain which, erroneously, has been considered rheumatic, neuralgic or neuritic. A painful flatfoot having a shortened gastrocnemius may often be relieved by restoring normal movement to the ankle joint, even without any further attempt at restoring the arch. When a neglected Shaffer's foot has resulted in a flatfoot an artificial arch alone can not be expected to cure. The prolonged use of steel arches is seldom necessary and must be productive of atrophy of the small muscles, ligaments and fasciæ of the foot.

The apparatus in general use for stretching the posttibial muscles is the traction shoe devised by Dr. Shaffer and presented to the profession about twenty-five years ago. With this machine any desired force can be applied. From a therapeutic standpoint it is unqualifiedly successful. The force is the resultant of two forces, one applied in an antero-posterior direction through a .strap passing behind the heel and called the heel-strap, and the other a force applied through a strap passing over the neck of the astragalus and called the astragalar strap. This resultant pulls the calcaneum downward and forward in an arch whose radii are from a center passing through the astragalus just below the external malleolus; the traverse axis of the ankle joint. In very resistant cases, however, the force exerted by the astragalar strap is such as to cause considerable discomfort

In the apparatus presented herewith the astragalar strap is not brought into use until the gastrocnemius is well stretched, and then a momentary hyperextension is obtained by the use of an antero-posterior ratchet exactly similar to the one in Shaffer's shoe.

The machine consists of two lateral bars, a stand and a foot-piece. The lateral bars A, A, are joined at their distal ends in a $U$ shape piece. Their proximal ends are bent so as to pass beneath the chair, where they are secured by a draw-pin which passes through a socket

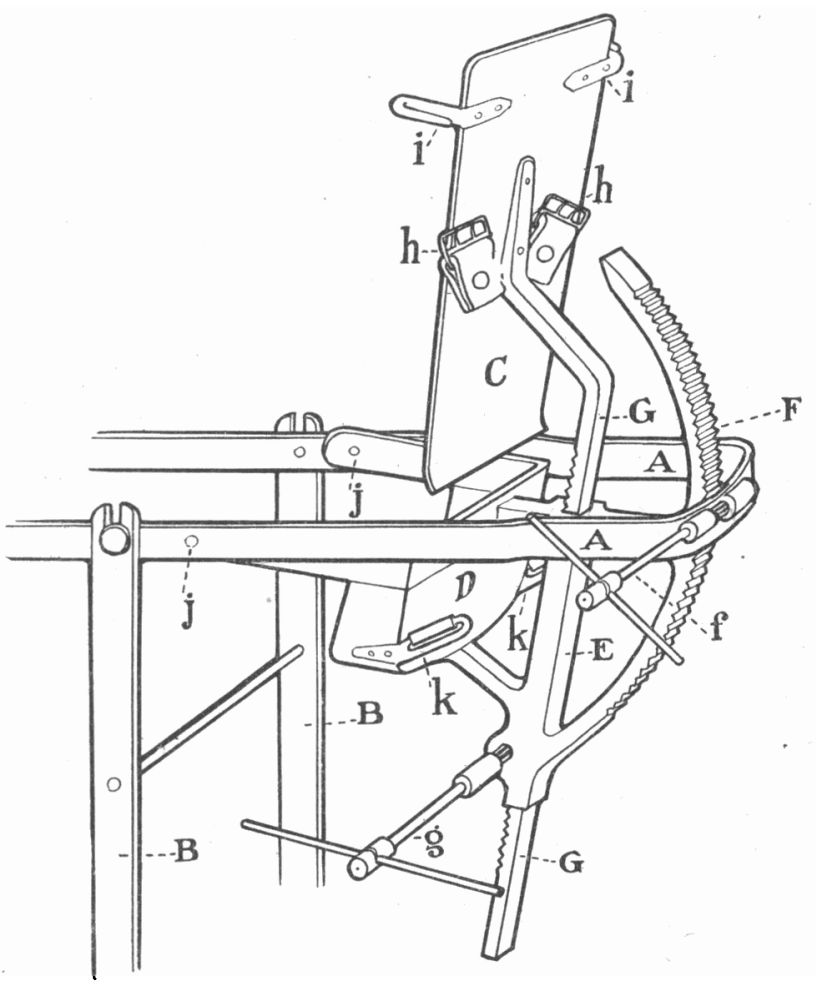

Figure 2 .

screwed to the bottom of the chair-seat. The continuity of these lateral bars is interrupted just below the calf-band $\mathrm{M}$, and the ends, overlapping, are held by thumb screws. Thus these bars may be shortened or lengthened as desired. The stand B, B supports the machine. It is detachable. The foot-piece consists of a foot-plate $\mathrm{C}$ and a heel-cup $\mathrm{D}$. The foot-plate is joined to the heel-cup through the ratchet bar $G$, passing through the sheath $E$, which is secured to the bottom of the heel-cup. The entire foot-piece is riveted to the lateral wars at $j, j$, and by means of the ratchet $F$ can be moved about the axis passing through $j, j$. The arc of the ratehet $\mathrm{F}$ is constructed on the radius $\mathrm{jF}$.

To the bottom of the foot-plate are riveted two buckles $h$, h, which secure the heel-strap. This strap passes bchind the heel, forward on both sides of the foot, over the hangers i, i, projecting upward from the front of the foot-plate and backward to the buckles. On each side of the heel-cup are pullevs, $k, k$, through which the astragalar $\mathbf{S}$ is roven. The strap crosses over the ankle opposite the head of the astragalus, from one pulley to the other, and is doubled back and one end passed through the buckle sewed to the other end. 
The method of application is as follows: The patient is seated in the chair, the heel placed in the heel-cup D, and the lateral bars $A, A$ are adjusted by means of the thumb screws to the exact length of the limb with the leg fully extended. It is necessary that the axis of the ankle joint should pass through the axis of rotation of the foot-piece $j, j$. The heel-strap assists greatly in obtaining the alignment of these two axes. While fitting the apparatus the foot-piece should be plantar flexed to an angle affording an easy position for the foot. The last thing to be done is to fasten the strap of the calf band. This prevents the patient flexing the knee as the strain comes on the gastrocnemius.

Traction may now be started. Using the key $f$, the ratchet $F$ is made to pass forward and around the axis $j, j$. Everything on that foot-piece will move through ares whose radii extend from the common center. The foot is thus moved, forcibly, about the ankle joint. When the tautness of the tendo-Achilles, as ascertained by palpation and the sensation of the patient, warns one that full flexion is obtained, the astragalar strap is applied and a momentary hyperextension produced by means of the antero-posterior ratchet G. Those familiar with Shaffer's traction shoe will recognize in this part of the treatment the copy of that shoe. Furthermore, it should be mentioned that Dr. Shaffer, by means of a worm and screw, also altered the angle of flexion and extension of the foot-pice.

\section{A NEW PRINCIPLE IN IRRIGATION OF THE URETHRA WITH INSTRUMENT.}

$$
\begin{aligned}
& \text { J. H. BACON, B.S., M.D. } \\
& \text { PEORYA, ILL. }
\end{aligned}
$$

I have been using a new principle in the treatment of gonorrlica, and the results seem to me to be good. The difference between my method of treating gonorrhea and that of those who use irrigations is not medicinal but mechanical. Instead of using only the force of pressure from gravity for irrigation of the urethra, I have added a bulb to the irrigation tube, and by intermittent pressing obtain an intermittent or rather a rhythmic pressure. The value of this kind of pressure over simple pressure was brought to my mind by seeing the blood vessels pulsate in the thigh of one of my patients while I was irrigating and remembering that the blood will not continue to circulate if the pressure is steady. It is only when the pressure is rhythmic that the blood will pass through the capillaries. Now. if we use Nature's method we must use rhythmic pressure in irrigating if we wish to have the fluid penetrate deeply into the lining membrane of the cavity irrigated. If the urethra were a flat surface the treatment would be simple and cleanliness could easily be maintained, but being an elastic tube it is more difficult to give it the very essential cleaning.

I use a quart glass fountain that can be raised or lowered at will. At the bottom it is connected with six feet of rubber tubing. Into this tubing about one foot and a half from the distal end is fitted a valveless rubber bulb the size of an atomizer bulb. A long, blunt glass nozzle is attached to the distal end of the tube, over which a half of a hollow rubber ball is placed in such a manner as to protect the hands of the operator from escaping fluid returning from the urethra. The bulb is used without valves so that the pressure used can not rise much above the fountain head without being regurgitated into the fountain. This protects the bladder from being invaded if the fountain is not raised more than two to two and one-half feet above the level of the urethra. All the apparatus can be readily and cheaply set up in the office, and yet be efficient.

When a patient presents himself in the early stage with a thick discharge showing the intracellular diplococci he is instructed to keep the bowels loose, drink freely of water, abstain from alcohol, coffee and spiced food, avoid excesses of all kinds and get abundance of sleep. Internally he is put on 15 grains of hexamethylenamine and 3 minims of oil of sandalwood three times a day. Sometimes I have given Lafayette's mixture in conjunction, with hexamethylenamine with good results. The urethra is irrigated four times a day with warm normal salt solution for the first week. The irrigating fluid is changed to $1: 5000$ potassium permanganate the second week and 1:1000 silver nitrate the third week. and this continued till cure is established. I hold the case cured if after one weeks' intermission no gonococci are found.

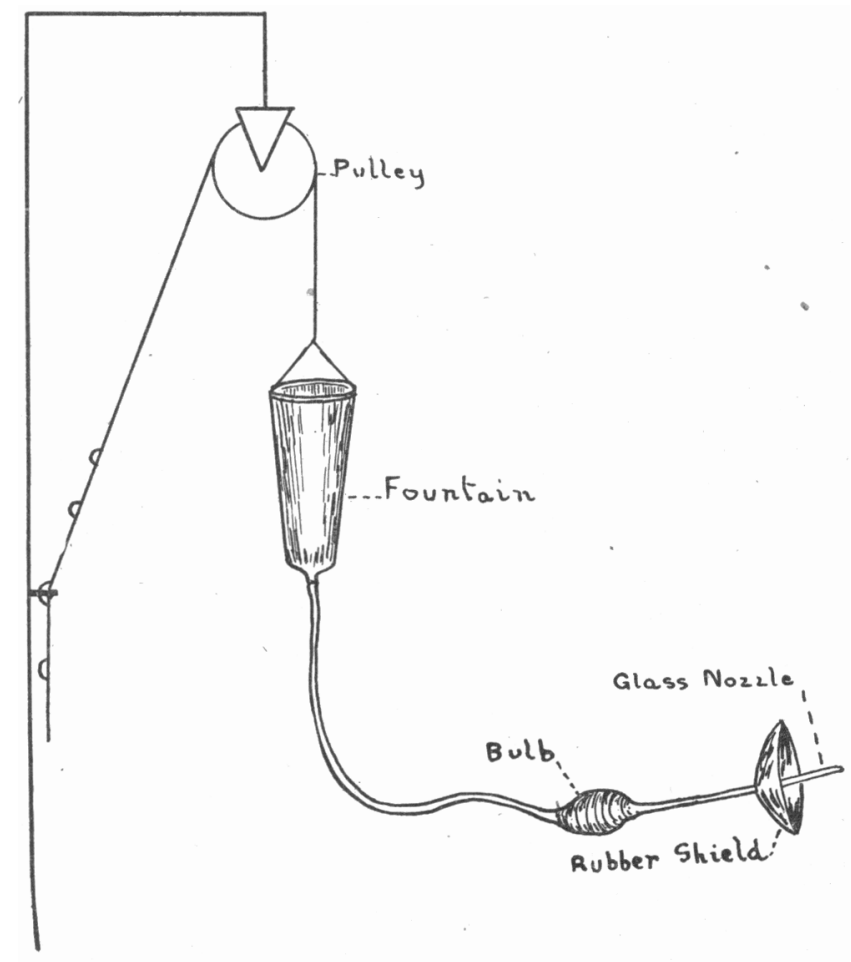

The irrigating fluid is heated to about 125 degrees Fahrenheit, the fountain maintained at two feet above the level of the urethra and the meatus and first half inch of canal washed out after the patient has urinated. The nozzle is then more firmly pressed into the urethra and the bulb pressed quickly and softly at the rate of about 80 to 90 to the minute. The urethra is seen to pulsate with each compression of the bulb at the same time there is some escape of fluid around the nozzle. The fluid penetrates deeply into the follicles and glands of the mucosa, the nidus of the infection and one can often see little yellowish white specks of pus that are jarred loose and swept out in this pulsating stream of fluid which would have remained in place for days and maintained the infection. In my experience the duration of an infection can be shortened from one to two weeks. This same principle of rhythmic pressure can be used with advantage, I believe, in abscess cavities, the rectum and colon, the pericardium and joints. 